\title{
ANÁLISE COMPARATIVA DA COOCORRÊNCIA DAS NOMINALIZAÇÕES EM -ÇÃO E EM -MENTO NO PORTUGUÊS BRASILEIRO E NO PORTUGUÊS EUROPEU
}

\author{
Ronaldo Rodrigues de Paula ${ }^{1}$ \\ Luis Filipe Lima e Silva²
}

\begin{abstract}
Resumo: O presente estudo utiliza o aporte teórico-metodológico dos estudos baseados em corpora (BIBER et al. 1998; McENERY; HARDIE, 2011) voltados para os estudos em variação linguística (LABOV, 1972; 1973). O objetivo deste trabalho consiste em analisar as ocorrências das nominalizações com sufixos em -ção e -mento dos seguintes pares mínimos Beneficiação/Beneficiamento, Congelação/Congelamento, Ordenação/Ordenamento, Povoação/Povoamento e Pronunciação/Pronunciamento no Corpus do Português (DAVIES; FERREIRA 2006) buscando determinar se elas apresentam o mesmo valor de verdade ou se apontam para diferentes direcionamentos semânticos, bem como se mostram indícios de variação e/ou mudança por meio da análise de colocados, de linhas de concordância e da distribuição diacrônica nos períodos distais do corpus. Os resultados mostraram que nem todas as nominalizações apresentaram o mesmo valor de verdade. Em alguns casos, elas eram mais comumente usadas para se referir a semas diferentes do verbo, ou em sentido metafórico e ainda registrou-se um claro quadro de mudança para um dos casos analisados.
\end{abstract}

Palavras-chave: Linguística de Corpus, Variação Linguística, Corpus do Português, Nominalizações em -ção e mento.

\section{Introdução}

As ferramentas da Linguística de Corpus tem um robusto potencial para análise de vários fenômenos da linguagem (BIBER et al. 1998; McENERY; HARDIE, 2011). Diferentes campos da linguística são contemplados pela aplicação de seus pressupostos teóricos e de seu instrumental metodológico, desde estudos mais estruturais visando entender e descrever melhor aspectos gramaticais e gramático-lexicais (DE HAAN, 1989; HUNSTON; FRANCIS, 2000; BIBER, 2005, entre outros) até estudos dedicados a compreender aspectos mais abrangentes da linguagem,

\footnotetext{
${ }^{1}$ Doutorado em linguística teórica e descritiva pela UFMG (2020). Mestrado em linguística teórica e descritiva pela UFMG (2015). Licenciatura plena em Língua Portuguesa e suas Literaturas e Língua Inglesa e suas Literaturas pela UFSJ (2013).E-mail: ronaldorodriguesdepaula@gmail.com

${ }^{2}$ Doutorado (2020) e mestrado (2016) em linguística teórica e descritiva pela Universidade Federal de Minas Gerais (UFMG). Obteve o título de Bacharel (2013) pela mesma instituição. Tem interesse em sintaxe, gramaticalização e pragmática.E-mail: luisf.1397@gmail.com
} 
que envolvem estudos discursivos, elaboração de materiais didáticos, noções de equivalência e adequação em tradução etc. (BAKER, 1993; RÖMER, 2005; YANG; LI, 2020, entre outros).

Dentre as inúmeras potencialidades que a Linguística de Corpus permite explorar, estão os estudos lexicais. A análise da frequência de colocados, tipos de registro de ocorrência e das próprias linhas de concordância podem revelar preferências de uso de certas palavras ou expressões tidas como candidatas a pares intercambiáveis em significado. Estes estudos podem determinar em quais contextos essa equivalência ocorre e em quais outros uma forma é preferida no lugar da outra, fornecendo importantes informações semânticas sobre os itens em análise (CANTOS-GÓMEZ; ALMELA-SÁNCHEZ, 2018).

Tomando como ferramentas os recursos teórico-metodológicos dos estudos de corpora, tem-se como objetivo, neste trabalho, determinar se diferentes nominalizações coocorrentes de um mesmo verbo com sufixação em -ção e em -mento possuem o mesmo valor de verdade tanto no português brasileiro quanto no português europeu. Sobre o conceito de valor de verdade, Cook (2009) explica que

Tipicamente, dentro da semântica formal, o status semântico de uma
proposição é representado em termos de um objeto particular assinalado
àquela proposição - seu valor de verdade. Nos contextos clássicos padrão
os valores de verdade são verdadeiro e falso (eventualmente 0 e 1 atuam
como substitutos para o verdadeiro e falso em contextos matemáticos),
e a lógica não-padrão equivale a nada mais do que extender a classe de
valores de verdade para além desse dois iniciais. (....”3 (COOK, 2009, p. 183)

Nos estudos de variação linguística (TARALLO, 1986), comumente se diz que duas formas variantes têm o mesmo valor de verdade se essas variantes compartilham o mesmo valor semântico.

Os pares selecionados foram: Beneficiação/Beneficiamento, Congelação/ Congelamento, Ordenação/Ordenamento, Povoação/Povoamento e Pronunciação/ Pronunciamento. Como fonte de dados, foi utilizado o Corpus do Português (DAVIES; FERREIRA 2006). Caso os pares apresentassem o mesmo valor de verdade, procurou-se analisar como eles se distribuíam diacronicamente em busca de indícios de variação ou de mudança (LABOV, 1972; 1973) nos períodos distais disponíveis no corpus. Caso os pares não apresentassem o mesmo valor de verdade, procurou-se estabelecer se haveria um direcionamento em termos semânticos para a preferência entre uma ou outra nominalização.

O artigo está dividido da seguinte forma. Primeiramente, a metodologia será apresentada. Em seguida, a análise dos dados, e por fim, as considerações finais.

\footnotetext{
${ }^{3}$ Do original: "Typically, within formal semantics, the semantic status of a statement is represented in terms of a particular object assigned to that statement - its truth value. In standard classical contexts the truth values are the true and the false (sometimes 0 and 1 act as surrogates for the true and the false in mathematical contexts), and non-standard logics often amount to nothing more than extending the class of truth values beyond these initial two.(...)” (COOK, 2009, p.183. Tradução nossa)
} 


\section{Metodologia}

Os cinco pares mínimos escolhidos foram analisados na função diagrama do Corpus do Português. Esta função mostra como as palavras se distribuem no eixo do tempo, agrupando dados desde o século XIV até o século XX, bem como em diferentes registros cobertos pelo corpus (Acadêmico, Notícias, Ficção e Oral) em relação ao número total de ocorrências, e, mais importante, na frequência normalizada, ou seja, na frequência da palavra em busca por milhão de palavras. Por fim, essa função ainda faz um comparativo do termo em busca das ocorrências no Brasil e em Portugal no século XX, pelos mesmos critérios citados. Este recurso pode mostrar qual forma surgiu em primeiro lugar, se sua frequência aumentou ou diminuiu em relação à coocorrente, o que pode ser bastante relevante para revelar indícios de variação e mudança.

Para determinar se os pares compartilhavam o mesmo valor de verdade, foi utilizado o recurso Comparar. Este recurso faz uma análise de colocados (palavras vizinhas) dos termos analisados, elencando os mais prototípicos de cada um, bem como os que são comuns a eles. Portanto, este recurso pode oferecer boas pistas dos usos preferenciais de cada forma ou se eles compartilham semas comuns. Essa função permite uma seleção de critérios e cria uma classificação para os colocados, conforme é mostrado na Figura 1 e na Tabela 1 abaixo:

Figura 1: Seleção de parametros

MOSTRAR
LISTA
DIAGRAMA

Na figura acima, temos Palavra(s), que são os campos para busca e comparação dos termos em questão. O uso de colchetes busca todos os lemas (isto é, todas as flexões) das palavras analisadas. Logo em seguida, temos Colocados. Os campos logo a frente determinam a quantidade de colocados à esquerda e à direita respectivamente (neste trabalho foram fixados quatro e quarto para todas 
as buscas). Abaixo temos Categoria Gramatical, que pode filtrar os resultados de colocados por uma categoria gramatical escolhida (no caso deste trabalho, optou-se por analisar, inicialmente, apenas os substantivos). Em seguida, temos as Secções, campo que se delimita o século, região ou registro a ser comparado.

Tabela 1: Exemplo de distribuição da função Comparar.

Recorte com os 5 primeiros colocados para as palavras analisadas: Povoação e Povoamento

\begin{tabular}{|c|l|c|c|c|c|}
\hline WORD 1 (W1): POVOAÇÃO (4.88) & W1 & W2 & P1/P2 & SCORE \\
\hline 1 & WORD & 8 & 0 & 16.0 & 3.3 \\
\hline 2 & [COLONO] & 7 & 0 & 14.0 & 2.9 \\
\hline 3 & [CASA] & 6 & 0 & 12.0 & 2.5 \\
\hline 4 & [CONCELHO] & 6 & 0 & 12.0 & 2.5 \\
\hline 5 & [ALDEIA] & 6 & 0 & 12.0 & 2.5 \\
\hline WORD 2 & (W2): PONOATIMONIO] & & & & \\
\hline & WORD & W2 & W1 & P2/P1 & SCORE \\
\hline 1 & [AREA] & 3 & 0 & 6.0 & 29.3 \\
\hline 2 & [EPOCA] & 3 & 0 & 6.0 & 29.3 \\
\hline 3 & [REMONTA] & 3 & 0 & 6.0 & 29.3 \\
\hline 4 & [REGIÃO] & 4 & 1 & 4.0 & 19.5 \\
\hline 5 & [VESTIGIO] & 4 & 1 & 4.0 & 19.5 \\
\hline
\end{tabular}

Fonte: Corpus do Português

Na Tabela 1, são mostrados os cinco primeiros resultados gerados pela seleção de parâmetros mostrada na Figura 1. Temos em Word 1, a palavra Povoação, e em Word 2, a palavra Povoamento. Os índices que aparecem a frente de cada palavra entre parênteses se referem à proporção de ocorrências de uma palavra em relação a outra. Desta forma, para cada ocorrência de Povoamento, Povoação ocorre cerca de 5 vezes. Similarmente, para cada ocorrência de Povoação, Povoamento ocorre 0,20 vezes. Inicialmente, tomemos apenas a tabela com a palavra Povoação para exemplificar as relações. Na linha em azul, temos a coluna Word que representa os colocados encontrados e W1 e W2, que representam o número total de ocorrências daquele colocado em relação a Word 1 (Povoação) e a Word 2 (Povoamento). Logo em seguida, temos $\mathrm{P} 1 / \mathrm{P} 2$ que calcula a proporção de ocorrências do colocado em relação às palavras ${ }^{4}$. E, por último, o Score, que é a divisão desta proporção do colocado entre as palavras (P1/P2) pela proporção das ocorrências da Word 1 em relação a Word 2, isto é, o índice indicado entre parênteses ${ }^{5}$. A exibição dos resultados se dá do maior Score para o menor, quando selecionamos nos parâmetros ordenar por relevância (cf. Figura 1). O mesmo procedimento é feito da Word 2 em relação a Word 1.

\footnotetext{
${ }^{4}$ Quando o número de ocorrências de um colocado para uma das palavras é 0 , se estabeleceu calcular a proporção utilizando-se o valor 0,5. Assim se, por exemplo, para a Word 1 houve 8 ocorrências de determinado colocado e para Word 2 não houve ocorrências, o valor da Proporção é $16(8 / 0,5)$.

${ }^{5}$ Por exemplo, temos a proporção P1/P2 = 16 para o colocado [Colono]. A proporção de Word 1 (Povoação) para a Word 2 (Povoamento) é de 4,88. O Score deste colocado para a relação W1/W2 será então a divisão destes coeficientes: $16 / 4,88=3,3$.
} 
Como foi exposto, quanto maior o Score, mais representativo será o colocado para uma palavra em relação à outra e mais forte será a associação desta palavra com este colocado em questão. Para a metodologia neste estudo, considerou-se a análise de colocados de maior Score para determinar os usos mais distintivos de uma palavra em relação à outra. Para tentar determinar o valor de verdade, consideraram-se os colocados que ocorreram para ambas as palavras analisadas, conferindo pelas linhas de concordância e pelo contexto se, de fato, as duas nominalizações possuíam o mesmo uso e sentido. Observou-se também o score destes colocados comuns, pois mesmo se constatado que as palavras analisadas compartilhavam o mesmo valor de verdade, para determinado colocado, um Score muito elevado ou muito baixo poderia mostrar uma associação mais comum para uma forma e mais atípica para outra, respectivamente. Por isso foram desconsiderados os colocados com Score menor que 0,3 ou maior que 3 .

\section{Análise dos dados}

\subsection{Beneficiação vs. Beneficiamento}

O Gráfico 1 abaixo apresenta as secções $s 14$ a s20 representando as sincronias, Port representando Portugal, Bras representando Brasil e, logo em seguida, as abreviaturas dos registros do corpus: Acadêmico, Notícias, Ficção e Oral. Na segunda linha, está representado o número total de ocorrências em cada secção e na linha seguinte a frequência por milhão. Como consta no gráfico, a nominalização Beneficiação ocorreu apenas nos registros de Portugal, enquanto a nominalização Beneficiamento ocorreu apenas nos registros do Brasil, com quase todas as ocorrências se concentrando no século XX. Além dessa diferença diatópica, houve também grande diferença entre a distribuição do par entre os registros. Beneficiação figura quase proeminentemente no registro Notícias ( $94 \%$ das ocorrências), ao passo que Beneficiamento tem menos da metade das ocorrências neste registro (cerca de 38\%) e é mais comum no registro Acadêmico (cerca de 62\%). Essa distribuição preferencial em diferentes registros já aponta indícios de uso distinto entre as duas formas. 
Gráfico 1: Recurso Diagrama: Distribuição de Beneficiação (acima) e Beneficiamento (abaixo)

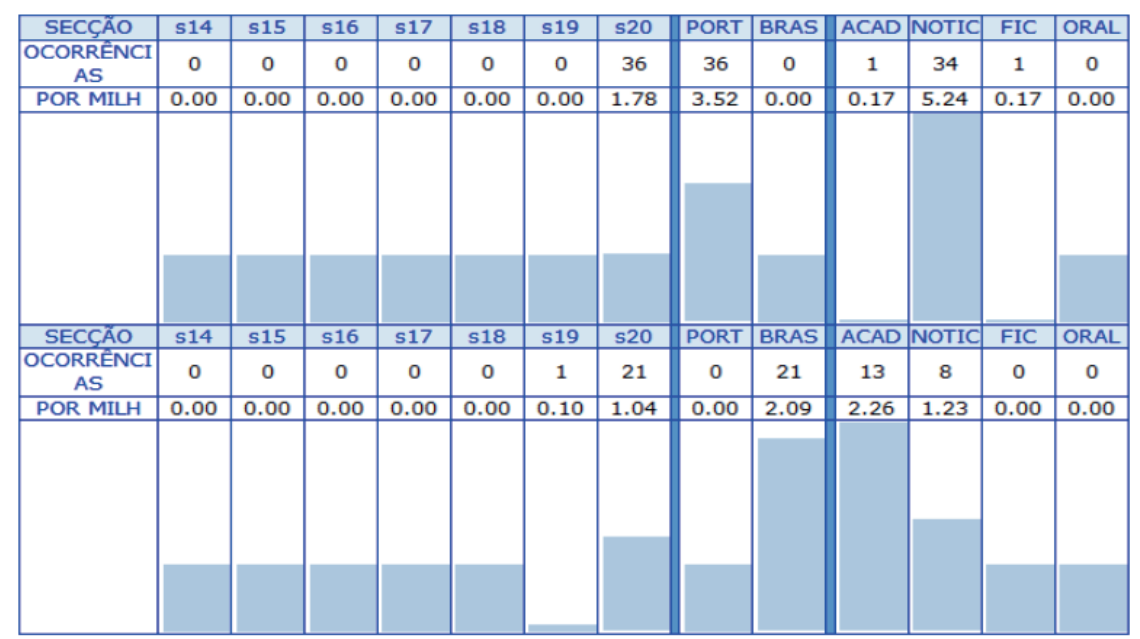

Fonte: Corpus do Português

Como no corpus as nominalizações não coocorreram numa mesma diatopia, serão analisados em seguida os colocados tentando estabelecer se Beneficamento, no Brasil, compartilha o mesmo valor de verdade de Beneficiação, em Portugal, ou se as nominalizações têm diferenças de uso na comparação geral.

\subsubsection{Análise de colocados (geral)}

A Tabela 2 aponta os colocados mais distintivos entre as duas formas.

Tabela 2: Recorte com os 10 colocados de maior Score: Beneficiação vs. Beneficiamento (geral)

\begin{tabular}{|c|c|c|c|c|c|}
\hline PALAVRA 1 (P1): BENEFICIAÇÃO (1.64) & PALAVRA & P2 & P1/P2 & SCORE \\
\hline 1 & [OBRA] & 12 & 0 & 24.0 & 14.7 \\
\hline 2 & [ESTRADA] & 9 & 0 & 18.0 & 11.0 \\
\hline 3 & [CONTO] & 3 & 0 & 6.0 & 3.7 \\
\hline 4 & [RECUPERAÇÃO] & 3 & 0 & 6.0 & 3.7 \\
\hline 5 & [REDE] & 2 & 0 & 4.0 & 2.4 \\
\hline 6 & [SANEAMENTO] & 2 & 0 & 4.0 & 2.4 \\
\hline 7 & [CONSTRUÇÃO] & 2 & 0 & 4.0 & 2.4 \\
\hline 8 & [ALTERNATIVA] & 2 & 0 & 4.0 & 2.4 \\
\hline 9 & [CONCLUSÃO] & 2 & 0 & 4.0 & 2.4 \\
\hline 10 & [MINISTRO] & 2 & 0 & 4.0 & 2.4 \\
\hline
\end{tabular}

\begin{tabular}{|c|c|c|c|c|c|}
\hline & PALAVRA & P2 & P1 & P2/P1 & SCORE \\
\hline 1 & [EXTRACÇÃO] & 5 & 0 & 10.0 & 16.4 \\
\hline 2 & [LAVRA] & 4 & 0 & 8.0 & 13.1 \\
\hline 3 & [INDÚSTRIA] & 2 & 0 & 4.0 & 6.5 \\
\hline 4 & [INDUSTRIALIZAÇÃO] & 2 & 0 & 4.0 & 6.5 \\
\hline 5 & [MINÉRIO] & 2 & 0 & 4.0 & 6.5 \\
\hline 6 & [PROJECTO] & 2 & 1 & 2.0 & 3.3 \\
\hline 7 & [RIO] & 1 & 0 & 2.0 & 3.3 \\
\hline 8 & [SEGMENTO] & 1 & 0 & 2.0 & 3.3 \\
\hline 9 & [SOJA] & 1 & 0 & 2.0 & 3.3 \\
\hline 10 & [SOLO] & 1 & 0 & 2.0 & 3.3 \\
\hline
\end{tabular}

Fonte: Corpus do Português 
Percebemos que os colocados com Score maior que dez (em verde) são [Obra] e [Estrada] para Beneficiação, e [Lavra] e [Extração] para Beneficiamento. Os colocados seguintes reforçam essa associação estabelecida pelos primeiros colocados. Analisemos, agora, os colocados comuns entre as duas formas:

Tabela 3: Colocados comuns: Beneficiamento vs Beneficiação (geral)

\begin{tabular}{|c|l|c|c|c|c|c|}
\multicolumn{7}{|c|}{ WORD 2 (W2): BENEFICIAMENTO (0.61) } \\
\hline 6 & WORD & [PROJECTO] & W2 & W1 & W2/W1 & SCORE \\
\hline 39 & [INSTALAÇÃO] & 1 & 1 & 2.0 & 3.3 \\
\hline
\end{tabular}

Fonte: Corpus do Português

Como consta na Tabela 3, apenas dois colocados comuns foram encontrados. O colocado [Projecto] tem um Score 3,3 para Beneficiamento em relação a Beneficiação. Suas linhas de concordância também foram analisadas, mas não seriam consideradas relevantes para determinar o valor de verdade. Mas, mesmo levando em conta os dois colocados, não foi constatado o mesmo valor de verdade para as nominalizações:

Tabela 4: Linhas de concordância dos colocados [Projecto] e [Instalação]

\begin{tabular}{|l|l|l|}
\hline 1 & $\begin{array}{l}\text { 19Ac:Br: } \\
\text { Lac:Thes }\end{array}$ & $\begin{array}{l}\text { geral avaliar a eficácia da aplicação dos procedimentos de AIA aos projetos de extração } \\
\text { e beneficiamento de minerais no Estado de São Paulo e sugerir modificações para seu } \\
\text { aperfeiçoamento. O }\end{array}$ \\
\hline$\underline{2}$ & $\begin{array}{l}\text { 19Ac:Br: } \\
\text { Lac:Thes }\end{array}$ & $\begin{array}{l}\text { da natureza do bem mineral, do porte da atividade, método de extração } \\
\text { e beneficiamento ou localização do projeto. Como decorrência desta orientação, a } \\
\text { mineração rapidamente assumiu a }\end{array}$ \\
\hline$\underline{1}$ & $\begin{array}{l}\text { 19N:Pt: } \\
\text { Beira }\end{array}$ & $\begin{array}{l}\text { com visita ao troço e conferência de imprensa para a apresentação pública } \\
\text { do projecto de beneficiação. Na altura, o vice-presidente da JAE deslocou-se a } \\
\text { Manteigas e garantiu que o }\end{array}$ \\
\hline$\underline{1}$ & $\begin{array}{l}\text { 19Ac:Br: } \\
\text { Lac:Thes }\end{array}$ & $\begin{array}{l}\text { edição da Resolução Conama no 1/86, as áreas licenciadas abrangiam somente } \\
\text { as instalações de beneficiamento do minério, enfocando tão-somente a poluição do ar } \\
\text { e da água. O despreparo }\end{array}$ \\
\hline$\underline{1}$ & $\begin{array}{l}\text { 19N:Pt: } \\
\text { Beira }\end{array}$ & $\begin{array}{l}\text { Clube de Futebol " Os Vilanovenses " foram contemplados com seis mil contos para } \\
\text { a beneficiação das instalações e construção das bancadas no campo do clube. No con }\end{array}$ \\
\hline
\end{tabular}

Fonte: Corpus do Português

Mesmo estes colocados comuns reforçam que Beneficiamento, no Brasil, e Beneficiação, em Portugal, têm usos diferentes e, portanto, diferentes valores de verdade. No Brasil, Beneficiamento se refere mais comumente a purificação, tratamento e refino de minerais. Este uso explica a grande quantidade do uso dessa nominalização no registro Acadêmico, provavelmente descrevendo técnicas e processos referentes a essa atividade. Por outro lado, Beneficiação, em Portugal, se refere mais comumente a reforma e reparo de vias. Este uso também explica a quase exclusividade deste sentido no registro Notícias, por ser um assunto de interesse público.

Porém, fora do corpus, foram encontradas instâncias de beneficiamento de vias no Brasil: 
Vianna conta ainda que essa era uma demanda antiga dessas pessoas e as conclusões foram comemoradas. O produtor rural Nivaldo Andrade Vieira aprovou os resultados e ele tem a convicção de que investir na área rural é um incentivo a mais para manter o agricultor no campo. "O beneficiamento de estradas, facilita o escoamento da produção agrícola, conserva veículos e equipamentos. Condições fundamentais para a nossa permanência aqui”, contou. ${ }^{6}$

Assim como foram encontras instâncias de beneficiação de minérios em Portugal:

Atividade: Extração e preparação de minérios não-ferrosos Compreende uma ou mais das seguintes atividades:

Extração e concentração de minérios de urânio e tório;

Extração, beneficiação (entre as quais, trituração, crivagem, ustulação) e concentração de minérios de cobre, estanho, volfrâmio, metais preciosos, alumínio (bauxite), chumbo, zinco, manganés, níquel, crómio, cobalto, molibdénio, tântalo, vanádio e outros minérios metálicos não ferrosos. ${ }^{7}$

Desta forma, fica atestado que Beneficiamento no Brasil tem os mesmos usos que Beneficiação em Portugal, porém no Corpus do Português um uso específico é mais registrado no Brasil (relativo ao tratamento, refino e purificação de minerais) e outro é mais registrado em Portugal (relativo ao reparo e reforma de vias).

\subsection{Congelação vs. Congelamento}

No Brasil, apenas a nominalização congelamento aparece nos registros. Em Portugal as duas formas coocorrem. Similarmente ao que ocorreu com o par Beneficiação vs. Beneficiamento, houve uma distribuição diferente nos registros. Congelação predomina no registro Acadêmico (cerca de 85\% das ocorrências), ao passo que Congelamento tem menos da metade das ocorrências neste registro (37,5\%), a maioria das ocorrências aparecendo no registro Notícias (62,5\%).

Como as duas formas coocorreram apenas em Portugal, a próxima seção analisará apenas os colocados na diatopia lusitana.

\footnotetext{
${ }^{6}$ Disponível em: <http://www.aracruz.es.gov.br/noticia/4843/>.

${ }^{7}$ Disponível em: <http://www.portaldaempresa.pt/cve/services/balcaodoempreendedor/Actividade. aspx?IdUnico=606>.
} 


\subsubsection{Análise de Colocados (Portugal)}

A Tabela 5 aponta os colocados mais distintivos entre as duas formas.

Tabela 5: Recorte com os 10 colocados de maior Score: Congelação vs. Congelamento (Portugal) WORD 1 (W1): CONGELAÇÃO $(1.10)$

\begin{tabular}{|c|c|c|c|c|c|}
\hline & WORD & W1 & W2 & $\mathrm{P} 1 / \mathrm{P2}$ & SCORE \\
\hline 1 & [PONTO] & 15 & 0 & 30.0 & 27.4 \\
\hline 2 & [TEMPERATURA] & 5 & 0 & 10.0 & 9.1 \\
\hline 3 & [DEPRESSÃO] & 4 & 0 & 8.0 & 7.3 \\
\hline 4 & [ALTERNÂNCIA] & 3 & 0 & 6.0 & 5.5 \\
\hline 5 & [DIMINUiçÃO] & 2 & 0 & 4.0 & 3.6 \\
\hline 6 & [GELO] & 5 & 2 & 2.5 & 2.3 \\
\hline 7 & [FUSÃO] & 4 & 2 & 2.0 & 1.8 \\
\hline 8 & [ALIMENTO] & 1 & 0 & 2.0 & 1.8 \\
\hline 9 & [ABAIXAMENTO] & 1 & 0 & 2.0 & 1.8 \\
\hline 10 & [АСТО] & 1 & 0 & 2.0 & 1.8 \\
\hline
\end{tabular}

\begin{tabular}{|c|l|c|c|c|c|}
\hline WORD 2 & (W2): CONGELAMENTO (0.91) & W2 & W1 & P2/P1 & SCORE \\
\hline & WORD & 2 & 0 & 4.0 & 4.4 \\
\hline 1 & [PALÁCiO] & 2 & 0 & 4.0 & 4.4 \\
\hline 3 & [PARECER] & 2 & 0 & 4.0 & 4.4 \\
\hline 4 & [ACELERADO] & 2 & 0 & 4.0 & 4.4 \\
\hline 5 & [SALÁRIO] & 2 & 0 & 4.0 & 4.4 \\
\hline 6 & [SANÇÃO] & 2 & 1 & 2.0 & 2.2 \\
\hline 7 & [DEGELO] & 1 & 0 & 2.0 & 2.2 \\
\hline 8 & [DESCONGELLMENTO] & 1 & 0 & 2.0 & 2.2 \\
\hline 9 & [FFECTUADO] & 1 & 0 & 2.0 & 2.2 \\
\hline 10 & [FINAL] & 1 & 0 & 2.0 & 2.2 \\
\hline
\end{tabular}

Fonte: Corpus do Português

Apenas um colocado com um Score maior que 10, na relação Congelação para Congelamento: [Ponto]. Este e os outros colocados mais distintivos de Congelação fazem clara alusão ao processo físico. Congelamento também apresentou colocados distintivos que fazem alusão ao processo físico, como [Degelo], porém também é associado a termos de outros campos semânticos como [Finança], [Salário], [Sanção]. A próxima tabela exibe os colocados comuns.

Tabela 6: Colocados comuns: Congelação vs. Congelamento (Portugal)

WORD 2 (W2): CONGELAMENTO (0.91)

\begin{tabular}{|c|l|c|c|c|c|}
\hline & WORD & W2 & W1 & P2/P1 & SCORE \\
\hline 6 & [DEGELO] & 2 & 1 & 2.0 & 2.2 \\
\hline 42 & {$[$ ÁGUA] } & 3 & 2 & 1.5 & 1.6 \\
\hline 43 & {$[$ FUSÃO] } & 2 & 4 & 0.5 & 0.5 \\
\hline 44 & {$[$ GELO] } & 2 & 5 & 0.4 & 0.4 \\
\hline
\end{tabular}

Fonte: Corpus do Português 
Vejamos algumas linhas de concordância:

Tabela 7: Linhas de concordância do colocado [Fusão]

\begin{tabular}{|c|c|c|}
\hline$\underline{1}$ & $\begin{array}{l}\text { 19Ac:Pt: } \\
\text { Enc }\end{array}$ & $\begin{array}{l}\text { meteorização física actua pelos processos de gelo e degelo (crioclastia - alternância } \\
\text { de congelação e fusão do gelo, em fendas e poros das rochas) e pela termoclastia }\end{array}$ \\
\hline$\underline{2}$ & $\begin{array}{l}\text { 19Ac:Pt: } \\
\text { Enc }\end{array}$ & $\begin{array}{l}\text { A meteorização física inclui os efeitos de gelo e degelo (fragmentação das rochas } \\
\text { pela congelação e fusão alternadas de água no interior de fendas) e a exfoliação, ou }\end{array}$ \\
\hline$\underline{3}$ & $\begin{array}{l}\text { 19Ac:Pt: } \\
\text { Enc }\end{array}$ & $\begin{array}{l}\text { processo em causa inclui o efeito de gelo e degelo (meteorização pela alternância } \\
\text { da congelação e fusão do gelo), os movimentos de massas (deslocamento de materiais } \\
\text { ao }\end{array}$ \\
\hline$\underline{4}$ & $\begin{array}{l}\text { 19Ac:Pt: } \\
\text { Enc }\end{array}$ & $\begin{array}{l}\text { sul da Inglaterra era periglaciar. A meteorização por gelo e degelo (alternância } \\
\text { de congelação e fusão do gelo em fendas de rochas) terá sido intensa, e terá }\end{array}$ \\
\hline$\underline{1}$ & $\begin{array}{l}\text { 19Ac:Pt: } \\
\text { Enc }\end{array}$ & $\begin{array}{l}\text { O processo de meteorização resulta provavelmente da combinação do efeito do gelo e } \\
\text { degelo (congelamento e fusão alternados da água e do gelo, em fendas nas rochas) e }\end{array}$ \\
\hline$\underline{2}$ & $\begin{array}{l}\text { 19Ac:Pt: } \\
\text { Enc }\end{array}$ & $\begin{array}{l}\text { leito para o bordo do glaciar, normalmente pelo efeito de gelo e degelo } \\
\text { (congelamento e fusão alternados da água e do gelo, em fendas das rochas); este }\end{array}$ \\
\hline
\end{tabular}

Fonte: Corpus do Português

Como ficou demonstrado pelas linhas de concordância, Congelação e Congelamento compartilham o mesmo valor de verdade, porém Congelamento parece se associar mais comumente a construções metafóricas no sentido de "paralisação”, principalmente em área econômica, o que explica a maior ocorrência do termo no registro Notícias. Por sua vez, Congelação se refere mais comumente ao estado físico, o que explica sua vinculação maior com o registro Acadêmico.

\subsection{Ordenamento vs Ordenação}

Em relação à distribuição por registro: Ordenamento tem a grande maioria das ocorrências nos registros Notícias e Acadêmico, enquanto Ordenação tem a grande maioria de ocorrências no registro Acadêmico. As duas formas coocorrem tanto em Portugal quanto no Brasil e serão analisadas nas próximas seções separadamente.

\subsubsection{Análise de Colocados (Portugal)}

Seguem abaixo a Tabela 8 com os colocados de maior Score para cada uma das nominalizações e a Tabela 9 com os colocados comuns: 
Tabela 8: Recorte com os 10 colocados de maior Score: Ordenamento vs. Ordenação (Portugal)

\begin{tabular}{|c|c|c|c|c|c|} 
WORD 1 & (W1): ORDENAMENTO (1.90) \\
\hline & WORD & W1 & W2 & P1/P2 & SCORE \\
\hline 1 & [TERRITORIO] & 59 & 1 & 59.0 & 31.1 \\
\hline 2 & [PLANO] & 23 & 0 & 46.0 & 24.3 \\
\hline 3 & [AMBIENTE] & 14 & 0 & 28.0 & 14.8 \\
\hline 4 & [LOCAL] & 10 & 0 & 20.0 & 10.5 \\
\hline 5 & [ADMINISTRAÇÃO] & 8 & 0 & 16.0 & 8.4 \\
\hline 6 & [GESTÃO] & 6 & 0 & 12.0 & 6.3 \\
\hline 7 & [PLANEAMENTO] & 6 & 0 & 12.0 & 6.3 \\
\hline 8 & [ÁREA] & 5 & 0 & 10.0 & 5.3 \\
\hline 9 & [GRUPO] & 4 & 0 & 8.0 & 4.2 \\
\hline 10 & [INSTRUMENTO] & 4 & 0 & 8.0 & 4.2 \\
\hline
\end{tabular}

\begin{tabular}{|c|l|c|c|c|c|}
\hline WORD 2 (W2): ORDENAÇÃO (0.53) & W2 & W1 & P2/P1 & SCORE \\
\hline 1 & WORD & 9 & 0 & 18.0 & 34.1 \\
\hline 2 & [MULHER] & 5 & 0 & 10.0 & 19.0 \\
\hline 3 & [METODO] & 3 & 0 & 6.0 & 11.4 \\
\hline 4 & [POMEM] & 3 & 0 & 6.0 & 11.4 \\
\hline 5 & [PADRE] & 2 & 0 & 4.0 & 7.6 \\
\hline 6 & [ORDENAVRA] & 2 & 0 & 4.0 & 7.6 \\
\hline 7 & [ORDENAÇÕO] & 2 & 0 & 4.0 & 7.6 \\
\hline 8 & [REAL] & 2 & 0 & 4.0 & 7.6 \\
\hline 9 & [LIVRO] & 2 & 0 & 4.0 & 7.6 \\
\hline 10 & [DIÁCONO] & 2 & 0 & 4.0 & 7.6 \\
\hline
\end{tabular}

Fonte: Corpus do Português

Tabela 9: Colocados comuns: Ordenamento vs. Ordenação (Portugal)

\begin{tabular}{|c|c|c|c|c|c|}
\hline & WORD & $\begin{array}{l}W \\
2\end{array}$ & $\begin{array}{c}W \\
1\end{array}$ & W2/W1 & SCORE \\
\hline 79 & [ECONOMIA] & 1 & 1 & 1.0 & 2.3 \\
\hline 80 & [BASE] & 1 & 1 & 1.0 & 2.3 \\
\hline 81 & [TERRITÓRIO] & 1 & 59 & 0.0 & 0.0 \\
\hline
\end{tabular}

Fonte: Corpus do Português

Inicialmente, já podemos destacar usos diferenciados para as nominalizações: Ordenamentoseassociouamuitos colocados comadenotaçãodeespaçofísico.Por suavez, Ordenação se associou mais à esfera religiosa, referente ao sacramento Ordem. [Mulher] é o colocado mais destacado, justamente pela polêmica da restrição deste Sacramento a homens apenas.

Dentre os colocados comuns, o único exemplo em que as nominalizações tiveram o mesmo valor de verdade ocorreu com o colocado [Território], conforme mostra a Tabela 10, porém como o Score deste colocado é muito elevado em favor de Ordenamento, não o consideramos relevante, é possível que o uso deste colocado para Ordenação tenha sido uma escolha idiossincrática e extremamente incomum. 
Tabela 10: Algumas linhas de concordância do colocado [Território]

\begin{tabular}{|l|l|l|}
$\underline{59}$ & $\begin{array}{l}\text { 19Ac:Pt: } \\
\text { Enc }\end{array}$ & $\begin{array}{l}\text { Governo Constitucional, também liderado por António Guterres, foi ministro do } \\
\text { Ambiente e do Ordenamento do Território entre } 1999 \text { e 2002. Em Abril de 2002, } \\
\text { depois da derrota }\end{array}$ \\
\hline 1 & $\begin{array}{l}\text { 19Ac:Pt: } \\
\text { Enc }\end{array}$ & $\begin{array}{l}\text { fiscal próprio, polícia autónoma (Ertzaintza), educação, saúde, } \\
\text { infraestruturas, ordenação do território. Economia O País Basco é uma antiga região } \\
\text { industrial, caracterizada ainda }\end{array}$ \\
\hline
\end{tabular}

Fonte: Corpus do Português

Pelos colocados mais distintivos Ordenamento está mais associado à organização, gestão e administração e Ordenação, além da associação ao sacramento Ordem, parece se associar mais a sequenciamento.

\subsubsection{Análise de Colocados (Brasil)}

Analisemos a Tabela 11 com os colocados de maior Score para cada uma das nominalizações:

Tabela 11: Recorte com os 10 colocados de maior Score: Ordenamento vs. Ordenação (Brasil)

\begin{tabular}{|c|l|c|c|c|c|}
\hline WORD & (W1): ORDENAMENTO (0.65) & W1 & W2 & P1/P2 & SCORE \\
\hline 1 & WORD & 5 & 0 & 10.0 & 15.3 \\
\hline 2 & [MUNICIPIO] & 3 & 0 & 6.0 & 9.2 \\
\hline 3 & [NORMA] & 2 & 0 & 4.0 & 6.1 \\
\hline 4 & [CULTURA] & 2 & 0 & 4.0 & 6.1 \\
\hline 5 & [FONSELHO] & 2 & 0 & 4.0 & 6.1 \\
\hline 6 & [GESTÃO] & 2 & 0 & 4.0 & 6.1 \\
\hline 7 & [INCORPORAÇÃO] & 2 & 0 & 4.0 & 6.1 \\
\hline 8 & [NIVEL] & 2 & 0 & 4.0 & 6.1 \\
\hline 9 & [SOCIEDADE] & 2 & 0 & 4.0 & 6.1 \\
\hline 10 & [MUDANÇA] & 2 & 1 & 2.0 & 3.1 \\
\hline
\end{tabular}

\begin{tabular}{|c|l|c|c|c|c|}
\hline WORD 2 & (W2): ORDENAÇÄO (1.53) & W2 & W1 & P2/P1 & SCORE \\
\hline & WORD & 3 & 0 & 6.0 & 3.9 \\
\hline 1 & [EFEITO] & 3 & 0 & 6.0 & 3.9 \\
\hline 2 & [CORRESPONDÊNCIA] & 3 & 0 & 6.0 & 3.9 \\
\hline 3 & [PALAVRA] & 3 & 0 & 6.0 & 3.9 \\
\hline 4 & [PARTE] & 3 & 0 & 6.0 & 3.9 \\
\hline 5 & [RESULTADO] & 3 & 0 & 6.0 & 3.9 \\
\hline 6 & [VARIAÇÃO] & 2 & 0 & 4.0 & 2.6 \\
\hline 7 & [PADRE] & 2 & 0 & 4.0 & 2.6 \\
\hline 8 & [USO] & 2 & 0 & 4.0 & 2.6 \\
\hline 9 & [PROBLEMA] & 2 & 0 & 4.0 & 2.6 \\
\hline 10 & [PROPOSTA] & \\
\hline & & & & \\
\hline
\end{tabular}

Fonte: Corpus do Português

Similarmente aos resultados de Portugal, Ordenamento parece se associar mais a questões de organização, gestão e administração. Ordenação parece se associar mais comumente a sequenciamento. Também houve ocorrências de colocados fazendo menção ao sacramento Ordem, porém não de forma tão destacada quanto em Portugal. 
Tabela 12: Colocados comuns: Ordenamento vs. Ordenação (Brasil)

\begin{tabular}{|c|c|c|c|c|c|}
\hline & WORD & $\begin{array}{l}w \\
2\end{array}$ & $\begin{array}{c}W \\
1\end{array}$ & $\begin{array}{c}\text { W2/W } \\
1\end{array}$ & SCORE \\
\hline $\begin{array}{l}1 \\
7\end{array}$ & [ANÁLISE] & 3 & 1 & 3.0 & 2.0 \\
\hline $\begin{array}{l}6 \\
9\end{array}$ & [VERIFICAÇÄO] & 1 & 1 & 1.0 & 0.7 \\
\hline $\begin{array}{l}7 \\
0\end{array}$ & [FACTO] & 1 & 1 & 1.0 & 0.7 \\
\hline $\begin{array}{l}7 \\
1\end{array}$ & [MUDANড̧A] & 1 & 2 & 0.5 & 0.3 \\
\hline
\end{tabular}

Fonte: Corpus do Português

Os colocados comuns não apresentaram o mesmo valor de verdade, com exceção, talvez, apenas no colocado apresentado na Tabela 13, mesmo assim, a correspondência não é muito clara.

Tabela 13: Linhas de concordância do colocado [Verificação]

\begin{tabular}{|l|l|l|}
\hline 1 & $\begin{array}{l}\text { 19Ac:Br } \\
\vdots \\
\text { Lac:The } \\
\text { s }\end{array}$ & $\begin{array}{l}\text { dez operações que convertem dados em informação, quais sejam: } \\
\text { captura; verificação; } \text { ordenamento; condensamento; cálculo; armazenamento; } \\
\text { recuperação; reproduç }\end{array}$ \\
\hline 1 & $\begin{array}{l}19 \mathrm{Ac}: \mathrm{Br} \\
\vdots \\
\text { Lac:Mis } \\
\text { c }\end{array}$ & $\begin{array}{l}\text { realiza nenhum tipo de análise semântica das sentenças. A simples verificação de } \\
\text { co-ocorrência e ordenação de palavras não garante que duas sentenças tenham o mesm }\end{array}$ \\
\hline
\end{tabular}

Fonte: Corpus do Português

Em suma, as duas nominalizações têm usos diferentes e parecem se associar preferencialmente a diferentes semas do verbo. ${ }^{8}$

\subsection{Povoação vs. Povoamento}

A primeira diferença mais notável entre Povoação e Povoamento se reflete na distribuição bastante díspar entre os registros. A primeira se distribui mais homogeneamente entre os registros, ao passo que a última tem uma associação muito maior como o registro Acadêmico. As duas formas coocorreram tanto em Portugal quanto no Brasil.

\footnotetext{
${ }^{8}$ Uma rápida pesquisa substituindo a classe gramatical de substantivos para adjetivos no parâmetro de busca de colocados mostrou um outro uso muito destacado de Ordenamento tanto no Brasil quanto em Portugal. Essa nominalização se associou com Score de 9,5 em Portugal e de 61,3 no Brasil ao colocado [Jurídico], o que denota grande vinculação à ordem no sentido normativo.
} 


\subsubsection{Análise de Colocados (Portugal)}

A Tabela 14 mostra os colocados mais distintivos entre as duas formas:

Tabela 14: Recorte com os 10 colocados de maior Score: Povoação vs. Povoamento (Portugal) WORD 1 (W1): POVOAÇÃO (4.88)

\begin{tabular}{|c|l|c|c|c|c|}
\hline & WORD & W1 & W2 & P1/P2 & SCORE \\
\hline 1 & [COLONO] & 8 & 0 & 16.0 & 3.3 \\
\hline 3 & [CASA] & 7 & 0 & 14.0 & 2.9 \\
\hline 4 & [CONCELHO] & 6 & 0 & 12.0 & 2.5 \\
\hline 5 & [ALDEIA] & 6 & 0 & 12.0 & 2.5 \\
\hline 6 & [PATRIMÓNIO] & 6 & 0 & 12.0 & 2.5 \\
\hline 7 & [VILA] & 6 & 0 & 12.0 & 2.5 \\
\hline 8 & [RIBINHO] & 5 & 0 & 10.0 & 2.0 \\
\hline 9 & [RUA] & 5 & 0 & 10.0 & 2.0 \\
\hline 10 & [PEQUENO] & 5 & 0 & 10.0 & 2.0 \\
\hline & & 5 & 0 & 10.0 & 2.0 \\
\hline
\end{tabular}

WORD 2 (W2): POVOAMENTO (0.20)

\begin{tabular}{|c|c|c|c|c|c|}
\hline & WORD & W2 & W1 & $\mathrm{P} 2 / \mathrm{P} 1$ & SCORE \\
\hline 1 & [AREA] & 3 & 0 & 6.0 & 29.3 \\
\hline 2 & {$[E P O C A]$} & 3 & 0 & 6.0 & 29.3 \\
\hline 3 & [REMONTA] & 3 & 0 & 6.0 & 29.3 \\
\hline 4 & [REGIÃO] & 4 & 1 & 4.0 & 19.5 \\
\hline 5 & [VESTIGIO] & 4 & 1 & 4.0 & 19.5 \\
\hline 6 & [ARQUIPÉLAGO] & 2 & 0 & 4.0 & 19.5 \\
\hline 7 & [COLONIA] & 2 & 0 & 4.0 & 19.5 \\
\hline 8 & [COLONIZAÇÃO] & 2 & 0 & 4.0 & 19.5 \\
\hline 9 & [FORMA] & 2 & 0 & 4.0 & 19.5 \\
\hline 10 & [HECTARE] & 3 & 1 & 3.0 & 14.6 \\
\hline
\end{tabular}

Fonte: Corpus do Português

Os colocados mais distintivos de Povoação associa o termo mais comumente à própria comunidade ou cidade, ou mesmo aos habitantes de determinada região. Povoamento se refere ao processo de ocupação e crescimento populacional em determinada região. Apesar dessa distinção ocorreram muitos colocados comuns, como veremos na Tabela 15 abaixo. 
Tabela 15: Colocados comuns: Povoação vs. Povoamento (Portugal)

WORD 2 (W2): POVOAMENTO $(0.20)$

\begin{tabular}{|c|c|c|c|c|c|}
\hline & WORD & w2 & W1 & W2/W1 & SCORE \\
\hline 4 & [REGIÃO] & 4 & 1 & 4.0 & 19.5 \\
\hline 5 & [VESTIGIO] & 4 & 1 & 4.0 & 19.5 \\
\hline 10 & [HECTARE] & 3 & 1 & 3.0 & 14.6 \\
\hline 11 & [TERRITÓRIO] & 5 & 2 & 2.5 & 12.2 \\
\hline 12 & [INÍCIO] & 2 & 1 & 2.0 & 9.8 \\
\hline 56 & [MADEIRA] & 2 & 2 & 1.0 & 4.9 \\
\hline 57 & [TARDE] & 1 & 1 & 1.0 & 4.9 \\
\hline 58 & [PERSPECTIVA] & 1 & 1 & 1.0 & 4.9 \\
\hline 59 & [PARTE] & 1 & 1 & 1.0 & 4.9 \\
\hline 60 & [MORTE] & 1 & 1 & 1.0 & 4.9 \\
\hline 61 & [ESTABELECIMENTO] & 1 & 1 & 1.0 & 4.9 \\
\hline 62 & [ESTADO] & 1 & 1 & 1.0 & 4.9 \\
\hline 63 & [CASO] & 1 & 1 & 1.0 & 4.9 \\
\hline 64 & [ACTIVIDADE] & 1 & 1 & 1.0 & 4.9 \\
\hline 65 & [ILHA] & 4 & 5 & 0.8 & 3.9 \\
\hline 66 & [HISTÓRIA] & 11 & 22 & 0.5 & 2.4 \\
\hline 67 & [TERRA] & 1 & 2 & 0.5 & 2.4 \\
\hline 68 & {$[Z O N A]$} & 1 & 2 & 0.5 & 2.4 \\
\hline 69 & [SÉCULO] & 1 & 3 & 0.3 & 1.6 \\
\hline 70 & [ORIGEM] & 1 & 3 & 0.3 & 1.6 \\
\hline 71 & [íNDIO] & 1 & 3 & 0.3 & 1.6 \\
\hline 72 & [VOLTA] & 1 & 4 & 0.3 & 1.2 \\
\hline 73 & [LOCAL] & 1 & 5 & 0.2 & 1.0 \\
\hline
\end{tabular}

Fonte: Corpus do Português

Estes colocados não apresentaram o mesmo valor de verdade para as duas formas, ao contrário, reforçaram ainda mais a diferença de uso como veremos pelos exemplos na Tabela 16: 
Tabela 16: Algumas linhas de concordância do colocado [História]

\begin{tabular}{|l|l|l|}
\hline$\underline{6}$ & $\begin{array}{l}\text { 19Ac:Pt: } \\
\text { Enc }\end{array}$ & $\begin{array}{l}\text {; o pelourinho e a ponte românica, em Aveloso. história Meda é } \\
\text { uma povoação muito antiga, como o comprovam os vestígios de épocas remotas } \\
\text { existentes no município. }\end{array}$ \\
\hline$\underline{7}$ & $\begin{array}{l}\text { 19Ac:Pt: } \\
\text { Enc }\end{array}$ & $\begin{array}{l}\text { constitui o elemento mais significativo ao nível do património } \\
\text { natural. história Mértola é uma povoação muito antiga. Foi utilizada como porto } \\
\text { fluvial do tráfego mediterrânico, pelo menos, }\end{array}$ \\
\hline$\underline{8}$ & $\begin{array}{l}\text { 19Ac:Pt: } \\
\text { Enc }\end{array}$ & $\begin{array}{l}\text { Pilar, em Barqueiros, a Quinta de Santiago, em Rede. história A povoação já existia } \\
\text { nos inícios da nacionalidade. Recebeu foral outorgado por D. Afonso Henriques }\end{array}$ \\
\hline$\underline{6}$ & $\begin{array}{l}\text { Enc:Pt: } \\
\text { Enc }\end{array}$ & $\begin{array}{l}\text { O litoral atlântico constitui o elemento mais significativo do património } \\
\text { natural. história O povoamento remonta a épocas entre a Idade da Pedra e a do } \\
\text { Bronze. Aqui se }\end{array}$ \\
\hline$\underline{7}$ & $\begin{array}{l}\text { 19Ac:Pt: } \\
\text { Enc }\end{array}$ & $\begin{array}{l}\text { rio Douro é o elemento mais significativo do património natural. história A origem } \\
\text { do povoamento é desconhecida. No século VIII estava ocupada pelos mouros. } \\
\text { Recebeu carta de foro }\end{array}$ \\
\hline$\underline{8}$ & $\begin{array}{l}\text { 19Ac:Pt: } \\
\text { Enc }\end{array}$ & $\begin{array}{l}\text { e o rio Sabor constituem os elementos mais significativos do património } \\
\text { natural. história De povoamento muito remoto, foi ocupada pelos mouros que lhe } \\
\text { deixaram o topónimo. Em 1273 }\end{array}$ \\
\hline
\end{tabular}

Fonte: Corpus do Português

\subsubsection{Análise de Colocados (Brasil)}

Analisemos a tabela abaixo:

Tabela 17: Recorte com os 10 colocados de maior Score: Povoação vs. Povoamento (Brasil)

\begin{tabular}{|c|c|c|c|c|c|}
\hline & WORD & W1 & W2 & $\mathrm{P} 1 / \mathrm{P} 2$ & SCORE \\
\hline 1 & [SERTÃO] & 2 & 0 & 4.0 & 4.7 \\
\hline 2 & [MOVIMENTO] & 2 & 0 & 4.0 & 4.7 \\
\hline 3 & [VIAGEM] & 2 & 0 & 4.0 & 4.7 \\
\hline 4 & [INTERIOR] & 2 & 1 & 2.0 & 2.3 \\
\hline 5 & [INVESTIDA] & 1 & 0 & 2.0 & 2.3 \\
\hline 6 & [LÉGUA] & 1 & 0 & 2.0 & 2.3 \\
\hline 7 & [LUZ] & 1 & 0 & 2.0 & 2.3 \\
\hline 8 & [MOCAMBO] & 1 & 0 & 2.0 & 2.3 \\
\hline 9 & [PASSAGEM] & 1 & 0 & 2.0 & 2.3 \\
\hline 10 & [PLANTAÇÃO] & 1 & 0 & 2.0 & 2.3 \\
\hline
\end{tabular}

\begin{tabular}{|c|l|c|c|c|c|}
\hline WORD 2 (W2): POVOAMENTO (1.17) & W2 & W1 & P2/P1 & SCORE \\
\hline 1 & WORD & 3 & 0 & 6.0 & 5.1 \\
\hline 2 & [EXPANSÃO] & 3 & 0 & 6.0 & 5.1 \\
\hline 3 & [ESTADO] & 3 & 0 & 6.0 & 5.1 \\
\hline 4 & [SÉCULO] & 5 & 1 & 5.0 & 4.3 \\
\hline 5 & [PROCESSO] & 8 & 2 & 4.0 & 3.4 \\
\hline 6 & [REGIÃO] & 2 & 0 & 4.0 & 3.4 \\
\hline 7 & [SANEAMENTO] & 2 & 0 & 4.0 & 3.4 \\
\hline 8 & [HISTÓRIA] & 1 & 0 & 2.0 & 1.7 \\
\hline 9 & [INSTANTÂNEO] & 1 & 0 & 2.0 & 1.7 \\
\hline 10 & [INSTRUÇÃO] & 1 & 0 & 2.0 & 1.7 \\
\hline
\end{tabular}

Fonte: Corpus do Português 
Os resultados no Brasil mostraram uma organização bastante similar a Portugal: Povoação se refere à comunidade ou aos habitantes, enquanto Povoamento se refere a ocupação ou crescimento populacional. No Brasil, houve menos colocados comuns:

Tabela 18: Colocados comuns: Povoação vs. Povoamento (Brasil)

\begin{tabular}{|l|l|l|l|l|l|}
\hline \multicolumn{9}{|c|}{ WORD 1 (W1): POVOAÇÃO } & $(0.86)$ \\
\hline & WORD & $\begin{array}{r}\text { W } \\
2\end{array}$ & $\begin{array}{c}\text { W2/W } \\
1\end{array}$ & SCORE \\
\hline 4 & 2 & 1 & 2.0 & 2.3 \\
\hline 4 & [INTERIOR] & 1 & 1 & 1.0 & 1.2 \\
\hline 6 & [TERRITÓRIO] & 1 & 1 & 1.0 & 1.2 \\
\hline 4 & [PARTE] & 1 & 1 & 1.0 & 1.2 \\
\hline 4 & [CAMPO] & 2 & 8 & 0.3 & 0.3 \\
\hline 8 & [REGIÃO] & 1 & 5 & 0.2 & 0.2 \\
\hline 9 & [PROCESSO] & & & \\
\hline 5 & & & & \\
\hline
\end{tabular}

Fonte: Corpus do Português

O único caso com o mesmo valor de verdade ocorreu com o colocado [Processo], (cf. Tabela 19) que tem um Score muito baixo para Povoação, portanto não foi considerado relevante.

Tabela 19: Algumas linhas de concordância do colocado [Processo]

\begin{tabular}{|l|l|l|}
\hline 1 & $\begin{array}{l}\text { 19Ac:Br: } \\
\text { Enc }\end{array}$ & $\begin{array}{l}\text { do atual Estado. Movimentos das Entradas e Bandeiras levaram ao início } \\
\text { do processo de povoação do território mineiro a partir do século XVI. ao } \\
\text { final do século seguinte surgem }\end{array}$ \\
\hline 1 & $\begin{array}{l}\text { 19Ac:Br: } \\
\text { Enc }\end{array}$ & $\begin{array}{l}\text { e do Pará foram iniciados a partir de } 1535 \text { pelos portugueses. } \\
\text { Tal processo de povoamento da região foi ineficaz perante novos } \\
\text { acontecimentos: com a incursão de invasores holandeses e }\end{array}$ \\
\hline
\end{tabular}

Fonte: Corpus do Português

\subsection{Pronunciação vs. Pronunciamento}

Há pouquíssimas ocorrências de Pronunciação tanto no Brasil quanto em Portugal no século XX. Essa forma foi bastante comum entre os séculos XVI e XIX, mas parece ter caído em desuso no século XX. Pronunciamento parece ter se iniciado no século XIX e aumentado bastante no século XX. Temos, portanto, um indício de mudança. Porém, temos que determinar se as formas compartilham o mesmo valor de verdade. 


\subsubsection{Análise de Colocados (Portugal)}

Colocados mais distintivos estão dispostos abaixo:

Tabela 20: Recorte com os colocados de maior Score: Pronunciação vs. Pronunciamento (Portugal)

\begin{tabular}{|c|c|c|c|c|c|c|}
\hline & WORD & W1 & W2 & \multicolumn{2}{|r|}{$\mathrm{P} 1 / \mathrm{P} 2$} & SCORE \\
\hline 1 & [CLAREZA] & 1 & 0 & \multicolumn{2}{|r|}{2.0} & 6.7 \\
\hline 2 & [SÍLABA] & 1 & 0 & \multicolumn{2}{|r|}{2.0} & 6.7 \\
\hline \multicolumn{7}{|c|}{ WORD 2 (W2): PRONUNCIAMENTO (3.33) } \\
\hline & WORD & & w2 & W1 & $\mathrm{P} 2 / \mathrm{P} 1$ & SCORE \\
\hline 1 & [MILITAR] & & 4 & 0 & 8.0 & 2.4 \\
\hline 2 & [ORIGEM] & & 2 & 0 & 4.0 & 1.2 \\
\hline 3 & [PAPA] & & 1 & 0 & 2.0 & 0.6 \\
\hline 4 & [QUEDA] & & 1 & 0 & 2.0 & 0.6 \\
\hline 5 & [REGENERAÇÃO] & & 1 & 0 & 2.0 & 0.6 \\
\hline 6 & [SIDONISTA] & & 1 & 0 & 2.0 & 0.6 \\
\hline 7 & [TARDE] & & 1 & 0 & 2.0 & 0.6 \\
\hline 8 & [CONJUNTURA] & & 1 & 0 & 2.0 & 0.6 \\
\hline 9 & [ESTADO] & & 1 & 0 & 2.0 & 0.6 \\
\hline 10 & [FORÇA] & & 1 & 0 & 2.0 & 0.6 \\
\hline
\end{tabular}

Fonte: Corpus do Português

O pequeno número de colocados para Pronunciação mostrado na Tabela 19 reflete o reduzido número de ocorrências no século XX. Os colocados apontam para uma distinção de uso entre as duas formas. Pronunciação se refere ao modo de se falar determinada palavra ou som, está se referindo a questões fonológicas. Pronunciamento se refere especificamente ao ato de proferir um discurso. Não houve colocados comuns.

\subsubsection{Análise de Colocados (Brasil)}

Como mostrado na Tabela 21, no Brasil os usos de Pronunciação e Pronunciamento são os mesmos de Portugal. Não houve colocados comuns.

Tabela 21: Recorte com os colocados de maior Score: Pronunciação vs. Pronunciamento (Brasil)

\begin{tabular}{|c|c|c|c|c|c|c|}
\hline & WORD & W1 & W2 & \multicolumn{2}{|c|}{ P1/P2 } & SCORE \\
\hline 1 & [LETRA] & 1 & 0 & \multicolumn{2}{|c|}{2.0} & 214.0 \\
\hline \multicolumn{7}{|c|}{ WORD 2 (W2): PRONUNCIAMENTO (107.00) } \\
\hline & WORD & & W2 & W1 & $\mathrm{P} 2 / \mathrm{P} 1$ & SCORE \\
\hline 1 & [PRESIDENTE] & & 8 & 0 & 16.0 & 0.1 \\
\hline 2 & [NAÇÃO] & & 4 & 0 & 8.0 & 0.1 \\
\hline 3 & [MINISTRO] & & 4 & 0 & 8.0 & 0.1 \\
\hline 4 & [LÍDER] & & 3 & 0 & 6.0 & 0.1 \\
\hline 5 & [TRIBUNAL] & & 3 & 0 & 6.0 & 0.1 \\
\hline 6 & [TELEVISÃO] & & 3 & 0 & 6.0 & 0.1 \\
\hline 7 & [SENADOR] & & 2 & 0 & 4.0 & 0.0 \\
\hline 8 & [PÚBLICO] & & 2 & 0 & 4.0 & 0.0 \\
\hline 9 & [QUINTA-FEIRA] & & 2 & 0 & 4.0 & 0.0 \\
\hline 10 & [REITOR] & & 2 & 0 & 4.0 & 0.0 \\
\hline
\end{tabular}

Fonte: Corpus do Português

O número reduzido de ocorrências de Pronunciação no século XX mostrou que esta forma não tem o mesmo valor de verdade de Pronunciamento neste século. 
Porém, achou-se frutífero analisar os colocados destas nominalizações no século XIX, quando havia um número bem maior de ocorrências da primeira forma. A Tabela 21 mostra os colocados mais distintivos das nominalizações no século XIX.

Tabela 22: Recorte com os 10 colocados de maior Score: Pronunciação vs. Pronunciamento (Séc.: XIX)

\begin{tabular}{|c|c|c|c|c|c|}
\hline & WORD & W1 & W2 & $\mathrm{P} 1 / \mathrm{P} 2$ & SCORE \\
\hline 1 & [ORADOR] & 8 & 0 & 16.0 & 5.8 \\
\hline 2 & [CAPITULO] & 6 & 0 & 12.0 & 4.4 \\
\hline 3 & $[A C C ̧ A ̃ O)]$ & 4 & 0 & 8.0 & 2.9 \\
\hline 4 & [COORDENADO] & 4 & 0 & 8.0 & 2.9 \\
\hline 5 & [CORTESÃO] & 4 & 0 & 8.0 & 2.9 \\
\hline 6 & [DECLAMAÇÃO] & 4 & 0 & 8.0 & 2.9 \\
\hline 7 & [ELOCUÇÃO] & 4 & 0 & 8.0 & 2.9 \\
\hline 8 & [GESTO] & 4 & 0 & 8.0 & 2.9 \\
\hline 9 & [MEMÓRIA] & 2 & 0 & 4.0 & 1.5 \\
\hline 10 & [VICIOSA] & 2 & 0 & 4.0 & 1.5 \\
\hline
\end{tabular}

\begin{tabular}{|c|l|l|l|l|l|}
\hline WORD 2 & (W2): PRONUNCIAMENTO (0.37) \\
\hline & WORD & W2 & W1 & P2/P1 & SCORE \\
\hline 1 & [DUVIDA] & 2 & 0 & 4.0 & 10.9 \\
\hline 2 & [EFEITO] & 2 & 0 & 4.0 & 10.9 \\
\hline 3 & [EXÉRCITO] & 1 & 0 & 2.0 & 5.5 \\
\hline 4 & [HISTÓRIA] & 1 & 0 & 2.0 & 5.5 \\
\hline 5 & [HOMEM] & 1 & 0 & 2.0 & 5.5 \\
\hline 6 & [IDEIA] & 1 & 0 & 2.0 & 5.5 \\
\hline 7 & [LEVANTE] & 1 & 0 & 2.0 & 5.5 \\
\hline 8 & [MARECHAL] & 1 & 0 & 2.0 & 5.5 \\
\hline 9 & [MONOTONIA] & 1 & 0 & 2.0 & 5.5 \\
\hline 10 & [PLUTOCRACIA] & 1 & 0 & 2.0 & 5.5 \\
\hline
\end{tabular}

Fonte: Corpus do Português

Como bem indicado pelos colocados [Orador] e [Declamação], a forma Pronunciação já fora usada, anteriormente ao século XX, com o sentido de se proferir um discurso, o que pode ser confirmado pelas linhas de concordância.

Um último teste feito foi a inclusão da forma Pronúncia na análise. Observemos o gráfico 2: 
Gráfico 2: Recurso Diagrama: Distribuição de Pronunciação (acima), Pronúncia (ao centro) e Pronunciamento (abaixo).

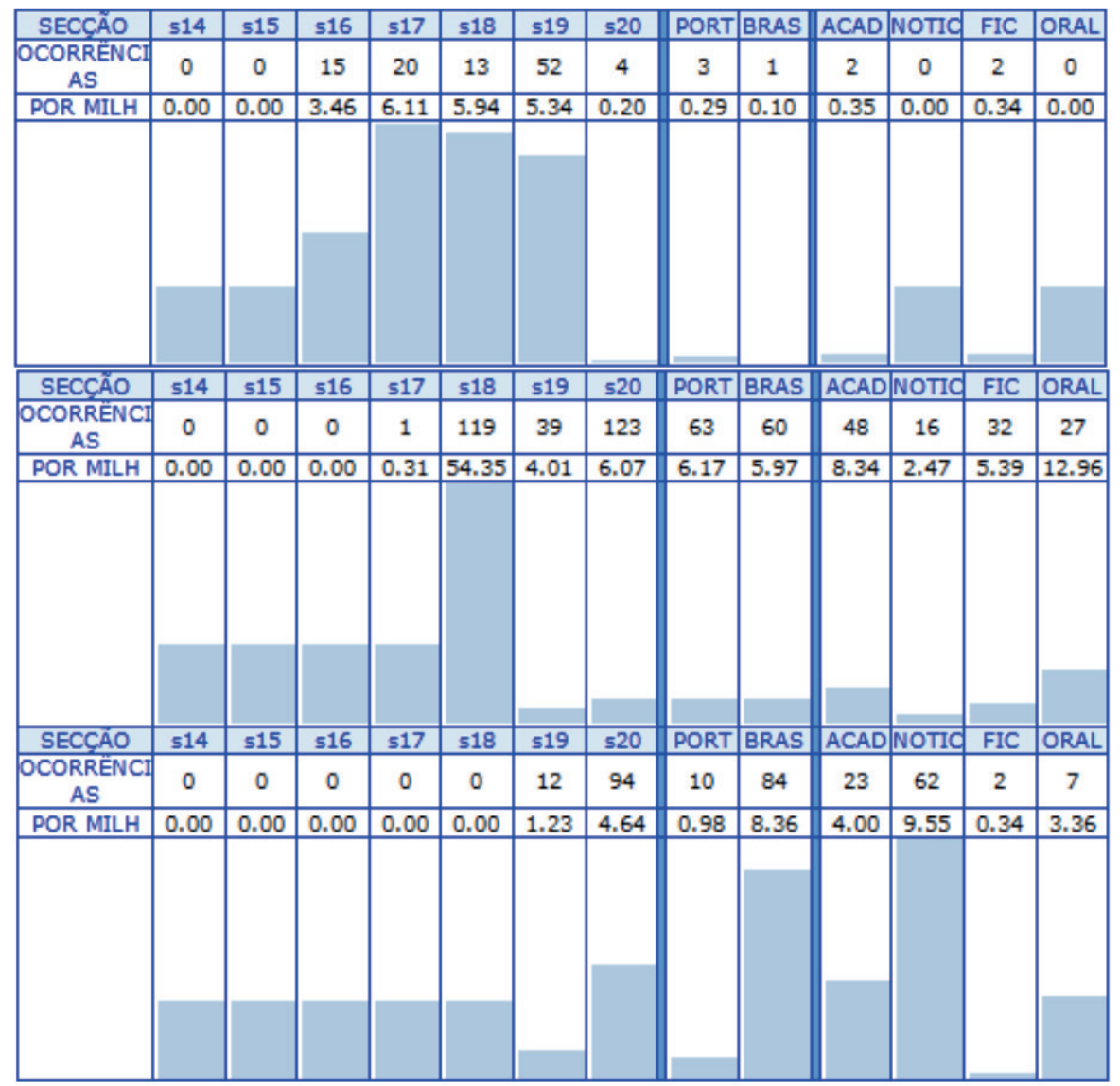

Fonte: Corpus do Português

Parece haver aí um quadro de mudança. A forma Pronunciação significava tanto dizer corretamente uma palavra, sílaba ou som, quanto proferir um discurso dos séculos XV ao XIX. Porém, no século XVII, surge a forma variante Pronúncia, que começa a concorrer com a forma inicial e aumenta consideravelmente no século XVIII. A forma Pronunciação mantém uma frequência por milhão similar durante os séculos XVII, XVIII, e XIX, (6,11, 5,94 e 5,34, respectivamente), pois, apesar do crescimento acentuado da forma Pronúncia no século XVIII, ela ainda mantinha o sentido de proferir um discurso. Porém, no século XIX, surge a forma Pronunciamento e suplanta completamente os usos de Pronunciação com este sentido no século XX. Pronunciação ainda tem registros escassos com sentido equivalente a Pronúncia no século XX, porém, pela comparação de frequência por milhão, é uma forma bastante atípica.

Como último teste, para garantir que a forma Pronúncia apresenta o mesmo valor de verdade de Pronunciação, na Tabela 23, destacam-se os colocados comuns destas duas formas no século XVIII. 
Tabela 23: Colocados comuns: Pronunciação vs. Pronúncia (Séc. XVIII)

\begin{tabular}{|l|l|l|l|l|l|}
\hline WORD 2 (W2): PRONÚNCIA (0.98) & W2 & W1 & W2/W1 & SCORE \\
\hline 9 & WORD & 6 & 2 & 3.0 & 3.0 \\
\hline 10 & [LINGUA] & 4 & 2 & 2.0 & 2.0 \\
\hline 11 & [VOGAL] & 2 & 1 & 2.0 & 2.0 \\
\hline 35 & [DIFERENÇA] & 6 & 4 & 1.5 & 1.5 \\
\hline 36 & [LETRA] & $Z$ & $Z$ & 1.0 & 1.0 \\
\hline 37 & [PALAVRA] & 1 & 1 & 1.0 & 1.0 \\
\hline 38 & [VEZ] & 1 & 1 & 1.0 & 1.0 \\
\hline 39 & [NSO] & 1 & 1 & 1.0 & 1.0 \\
\hline 40 & [DITONGOM] & 1 & 1 & 1.0 & 1.0 \\
\hline 41 & [VOZ] & 1 & 1 & 1.0 & 1.0 \\
\hline 42 & [NOME] & 3 & 4 & 0.8 & 0.8 \\
\hline 43 & [DIVERSIDADE] & 1 & 2 & 0.5 & 0.5 \\
\hline 44 & [LATINO] & 1 & 3 & 0.3 & 0.3 \\
\hline 45 & [ORTOGRAFIA] & 1 & 13 & 0.1 & 0.1 \\
\hline
\end{tabular}

Fonte: Corpus do Português

\section{Considerações Finais}

Este estudo mostrou que nem todas as coocorrências de nominalizações em -ção e -mento derivadas da mesma raiz verbal que ocorreram no Corpus do Português apresentaram o mesmo valor de verdade. Das formas com o mesmo valor, podemos deduzir que Beneficiar em Portugal privilegia a nominalização em -ção e no Brasil em -mento. Congelar apresenta apenas a forma em -mento no Brasil e as duas em Portugal, denotando o processo físico, porém apenas a forma em -mento com uso metafórico.

Das formas com valores de verdades diferentes, as nominalizações de ordenar tanto no Brasil quanto em Portugal tem associações preferenciais a certos campos específicos e diferentes semas. E tanto no Brasil quanto em Portugal, povoamento, em geral, se refere ao processo de crescimento populacional, enquanto povoação se refere à determinada comunidade ou a seus habitantes. E, por fim, a nominalização original para o verbo Pronunciar, isto é, Pronunciação, parece ter dado lugar a duas outras formas distintas, Pronúncia e Pronunciamento, para expressar seus diferentes semas. 


\title{
COMPARATIVE ANALYSIS OF CO-OCCURRENCE OF NOMINALIZATIONS WITH THE USE OF SUFFIXES -ÇÃO AND -MENTO IN BRAZILIAN PORTUGUESE AND EUROPEAN PORTUGUESE
}

\begin{abstract}
This study is built upon the theoretical and methodological contributions of the studies based on corpora (BIBER et al. 1998; McENERY; HARDIE, 2011) with special focus on studies within linguistic variation (LABOV, 1972; 1973). The main objective of this work is to analyze the occurrences of nominalizations in Portuguese with the use of the suffixes -ção and -mento of the following pairs: Beneficiação/ Beneficiamento, Congelação/ Congelamento, Ordenação/ Ordenamento, Povoação/ Povoamento and Pronunciação/ Pronunciamento in Corpus do Português (DAVIES; FERREIRA 2006). The main goal is to determine whether the pairs have the same truth value or if they point to different semantic values, as well as if they display signs of variation and/ or linguistic change via the analysis of Collocates, Concordance lines and diachronic distribution in the distal periods of the corpus. The analysis showed that not all pairs of nominalizations have the same truth value. In some cases, they were more commonly used to refer to different meanings of the verb, or for a metaphorical sense and there was a clear case of linguistic change in one of the pairs discussed.
\end{abstract}

Keywords: Corpus Linguistics, Linguistic Variation, Corpus do Português, Nominalization Suffixes -ção/-mento.

\section{Referências Bibliográficas}

BAKER, M. Corpus linguistics and translation studies. In: BAKER, M.; FRANCIS, G.; TOGNINI-BONELLI, E. (eds.). Text and technology. Amsterdam/Philadelphia: John Benjamins, 1993. p. 232-252.

BIBER, D. Corpus Linguistics and the Study of English Grammar.

Indonesian Journal of English Language Teaching, v. 1, n. 1, p. 1-22, 2005.

BIBER, D.; CONRAD, S.; REPPEN, R. Corpus Linguistics: Investigating Language Structure and Use. Cambridge: Cambridge University Press, 1998.

CANTOS-GÓMEZ, P.; ALMELA-SÁNCHEZ, M. (eds.). Lexical Collocation Analysis: Advances and Aplications. Cham: Springer, 2018.

COOK, R. T. What Is a Truth Value and How Mare Are There?

In: Studia Logica: An International Journal for Symbolic Logic, Vol. 92, No. 2, TruthValues. Part II, 2009 pp. 183-201

DE HAAN, P. Postmodifying clauses in the English noun phrase: a corpus-based study. Amsterdam: Rodopi, 1989.

DAVIES, M.; FERREIRA, M. Corpus do Português: 45 million words, 1300s-1900s. 2006. Disponível em: <https://www.corpusdoportugues.org>. Acesso em: 15 ago. 2020.

HUNSTON, S.; FRANCIS, G. Pattern Grammar: A corpus-driven approach to the lexical grammar of English. Amsterdam; Philadelphia: John Benjamins, 2000. 
LABOV, W. Sociolinguistic patterns. Philadelphia: University of Pennsylvania Press, 1972.

LABOV, W. The boundaries of words and their meanings. In: BAILEY, C. J. N., SHUY, R. W. (eds.). New ways of analysing variation in English. Washington: Georgetown University Press, 1973. p. 340-373.

McENERY, T.; HARDIE, A. Corpus Linguistics: Method, Theory and Practice. New York: Cambridge University Press, 2011.

RÖMER, U. Progressives, Patterns, Pedagogy: A corpus-driven approach to English progressive forms, functions, contexts and didactics. Amsterdam/ Philadelphia: John Benjamins, 2005.

TARALLO, F. A Pesquisa Sociolinguística. São Paulo: Ática, 1986.

YANG, B.; LI, W. (eds.). Corpus-based Approaches to Grammar, Media and Health Discourses: Systemic Functional and Other Perspectives. Cham: Springer, 2020. 